\title{
DDI Timeline
}

by Mary Vardigan ${ }^{1}$

\section{Abstract}

This timeline lists key developments and events in the history of the DDI, beginning with selected foundational developments that set the stage for DDI and facilitated its creation. Note that while many archives have played a significant role in DDI history, only the ones established early on are listed here.

1946

Roper Center established.

1960

Zentralarchiv established.

1962

Inter-university Consortium for Political [and Social] Research (ICPSR) established.

1964

Steinmetz Archive established.

1965-1968

MARC (Machine Readable Cataloging) standards developed at [U.S.] Library of Congress.
1974

International Association for Social Science Information Services and Technology (IASSIST) formed following the Conference on Data Archives and Program Library Services, held in conjunction with the 8th World Conference of Sociology in Toronto, Canada.

\section{4}

Report issued on "Standardization of Study Description Schemes and Classification of Indicators," a product of a meeting held in Copenhagen, Denmark, at the Danish Data Archives with nine archives from six countries in attendance.

\section{5}

IASSIST establishes Classification Action Group.

1976

Council of European Social Science Data Archives (CESSDA) established.

\section{8}

Anglo-American Cataloguing Rules, Second Edition (AACR2) published by the American Library Association (includes a new Chapter 9, for machine-readable data files).

1967

United Kingdom

Data Archive (UKDA)

established.

1967

\section{Selected foundational developments that set the stage for DDI}

OSIRIS (Organized Set

of Integrated Routines for Investigations with Statistics) statistical software package developed at University of Michigan, Institute for Social Research.

1968

First version of SPSS (Statistical Package for the Social Sciences) released.

1970

American Library Association appoints Subcommittee to Recommend Rules for Cataloging Machine-Readable Data Files.

\section{1}

Norwegian Social Science Data Service (NSD) established.

\section{9}

Sue Dodd publishes "Bibliographic References for Numeric Social Science Data Files: Suggested Guidelines." Journal of the American Society for Information Science, Volume 30, Issue 2, pp. 77-82, March 1979.

\section{0}

A Style Manual for Machine-readable Data Files and Their Documentation published by R.C. Roistacher with contributions from Sue Dodd, B.B. Noble, and Alice Robbin.

1982

Sue Dodd publishes Cataloging Machine-Readable Data Files. An Interpretive Manual. American Library Association, Chicago. 
1985

Sue Dodd and Ann M. Sandberg-Fox publish Cataloging

Microcomputer Files: A Manual of Interpretation for AACR 2.

1985

Fienberg et al. publish Sharing Research Data. Committee on National Statistics. Commission on Behavioral and Social Sciences and Education. National Research Council. Washington, DC: National Academies Press, 1985.

\section{5}

NSFNet, the precursor to the Internet, created.

1986

SGML (Standard Generalized Markup Language), descended from IBM's Generalized Markup Language (GML) developed in the 1960s, becomes an ISO standard.

1989

World Wide Web invented.

1989

International Federation of Library Associations (IFLA) recommends an International Standard Bibliographic Description for Computer Files (ISBD/CF).

1993

IASSIST forms Action Group for "Codebook Documentation of Social Science Data."

\section{3}

CESSDA holds seminar on "Variable Level Documentation" in Gothenburg, Sweden.

1994

Dublin Core Metadata Initiative established.

1995

First SGML Codebook Committee, constituted by ICPSR Director Richard Rockwell, meets in Quebec City, Canada. Members develop a draft list of codebook elements.

1996

First DDI specification prepared at University of Michigan Library. An SGML Document Type Definition (DTD) was produced by David Barber and John Brandt (University of Michigan), Ann Green (Yale University), and the DDI Committee.

1996

First Working Draft of XML (eXtensible Markup Language) published.

1997

U.S. National Science Foundation (NSF) funding received to enhance DDI and betatest it. This award (SBR-9617813) funded DDI development and ICPSR codebook digitization. Final Report ( $p d f$ )

1997

SGML DDI specification translated to XML.

This work was done by Jan Nielsen, Danish Data Archive.
1998

Committee meets in New Haven, CT.

Prepares for betatesting.

1999

Betatest of DDI DTD takes place (see related article for testers names).

2000

DDI Version 1 (DTD-based) published.

View Version 1 and successive iterations

2001

Formal DDI evaluation, funded by NSF, takes place.

Evaluators praise the effort

Evaluation Report

2001

Funding for DDI development received from Health Canada during 2001-2002.

Covers costs of meetings until Alliance established

2001

Working group on aggregate data meets in Voorburg, Netherlands. Group develops a proposal for DDI coverage of aggregate/tabular data

2001

First DDI Training held at IASSIST in Amsterdam, Netherlands. Bill Block and Wendy Thomas lead the workshop on "Creating DDI Compliant Codebooks"

2002

Bjorn Henrichsen, NSD, becomes Chair of the DDI Committee.

2002

Committee meets in Storrs, CT, to draft DDI Alliance charter. View the Charter

2003

Final meeting of original DDI Committee held in Washington, DC (see related article for members' names).

Minutes

2003

DDI Alliance established with Tom Piazza, UC-Berkeley, Chair. View "About the Specification" from original Alliance Web site, March 2007

2003

DDI Alliance Steering Committee meets for the first time. Minutes

2003

DDI 2 published.

DDI now covers aggregate data and geography

View Version 2 and successive iterations

DTD Version History 
2003

DDI Expert Committee meets for first time in Ann Arbor, MI. Committee discusses transition from DTD to Schemas; New working groups on Structural Reform and Substantive Issues formed Minutes

2004

DDI Expert Committee meets in Madison, WI.

Committee discusses requirements for Version 3

Minutes

Lifecycle Model

2005

Hans Jorgen Marker, DDA, becomes Chair of the DDI Expert

Committee ; Ron Nakao, Stanford, Vice Chair.

2005

DDI Expert Committee meets in Edinburgh, Scotland.

Committee ratifies life cycle model and DDI 3 begins to take shape Minutes

\section{6}

DDI Expert Committee meets in Ann Arbor, MI.

Committee approves the scope and timeline for Version 3 Minutes

2007

Public Review of DDI 3 takes place.

2007

DDI Expert Committee meets in Montreal, Canada.

Committee approves Candidate Draft of DDI 3

Minutes

2007

First DDI Training Workshop takes place at Schloss Dagstuhl in

Wadern, Germany.

Course description

2008

DDI 3 published as XML Schemas.

Version 3.0

2008

Expert Committee meets at IASSIST in Palo Alto, CA.

Plans for tools development discussed

Minutes

2008

DDI develops Best Practices for DDI 3.

Best Practices

2009

DDI Lifecycle 3.1 published.

Link to Specification

2009

First European DDI Users Conference (EDDI) held in Bonn, Germany. Program (PDF)
2009

DDI Expert Committee meets in Tampere, Finland.

Committee discusses tools and outreach to NSIs

Minutes (pdf)

2010

Chuck Humphrey, University of Alberta, becomes Chair of the DDI

Expert Committee; Mari Kleemola, FSD, Vice Chair.

2010

Second EDDI Conference held in Utrecht, Netherlands.

Program (pdf)

2010

DDI Developers Group meets for first time in Utrecht, Netherlands. View Minutes and Reports

2010

DDI Expert Committee meets in Ithaca, NY.

Rebranding DDI 2 and 3 as DDI Codebook and Lifecycle approved Minutes (pdf)

2011

External review of DDI takes place.

Review report

2011

DDI Alliance publishes first set of controlled vocabularies.

View vocabularies

2011

Expert Committee meets in Vancouver, Canada.

Results of external review discussed

Minutes (pdf)

2011

Third EDDI Conference held in Gothenburg, Sweden.

Program (pdf)

2011

DDI Alliance releases Tools Catalog.

View catalog

2011

DDI Alliance establishes agency registry.

View registry

2012

DDI Expert Committee Meets in Washington, DC.

Plans for a model-based specification discussed

Minutes (pdf)

2012

DDI Codebook 2.5 published as XML schemas.

Specification

2012

First Dagstuhl Workshop on model-based DDI held.

Description

Paper 
2012

Fourth EDDI Conference held in Bergen, Norway. Program

2013

DDI RDF Discovery and XKOS vocabularies published. Description

2013

First North American DDI Users Conference (NADDI) Held in Lawrence, KS.

Program

\section{3}

DDI Membership/Scientific Board Meets in Cologne, Germany. Transition to new governance structure discussed Minutes

\section{3}

First DDI Executive Board (successor to Steering Committee) meets with Gillian Nicoll, Australian Bureau of Statistics, as Chair and Ron Nakao, Stanford University, as Vice Chair.

View members and minutes

\section{3}

DDI "Sprints" launched to work on model-based DDI.

\section{More information}

\section{3}

Fifth EDDI Conference held in Paris, France.

\section{Program}

2014

DDI Lifecycle Version 3.2 published.

\section{References}

"About the [DDI] Specification." DDI Alliance Web Site (via the Internet Archive Wayback Machine): <http://web.archive.org/ web/20031112160748/www.icpsr.umich.edu/DDI/codebook/index. html> (Accessed February 12, 2014)

Block, Bill, Wendy Thomas, Robert Wozniak, and Joshua Buysse. (2001)

Creating DDI Compliant Codebooks: <http://www.google.com/url?s $a=t \& r c t=j \& q=\& e s r c=s \&$ source $=$ web $\& c d=2 \& c a d=r j a \& v e d=0 C D I Q F j A$ B\&url=http\%3A\%2F\%2Fwww.iassistdata.org\%2Fdownloads\%2Fddi_ workshop.pdf\&ei=rgGyUuiuEPPlyAGYsoD4CA\&usg=AFQjCN Foh-bgmplkK3YvY4RSk9m69bLflg\&sig2=gxmmc5iv18SqEkInRNFecQ $\& b v m=b v .58187178$, d.aWc $>$

Breckenhill, Inc. (2011) DDI Alliance External Review: Summary and Recommendations July 8, 2011. <http://www.ddialliance.org/sites/ default/files/DDI\%20Alliance\%20Review\%20Summary\%202011-0708.pdf>

DDI Alliance Agency Registry. <http://registry.ddialliance.org/> (Accessed February 12, 2014)

DDI Alliance Original Charter (via the Internet Archive Wayback Machine): <http://web.archive.org/web/20040502162842/www. icpsr.umich.edu/DDI/org/charter.html> (Accessed February 12, 2014)

DDI Best Practices for DDI 3. <http://www.ddialliance.org/resources/ publications/working/bestpractices> (Accessed February 12, 2014)
DDI [Codebook] DTD Version 1 and successive iterations. <http://ddialliance.cvs.sourceforge.net/viewvc/ddi-alliance/ddi/dtd/Version1. $\mathrm{dtd}$ ?view=log $>$ (Accessed February 12, 2014)

DDI [Codebook] DTD Version 2 and successive iterations: $<$ http:// ddi-alliance.cvs.sourceforge.net/ddi-alliance/ddi/dtd/> (Accessed February 12, 2014)

DDI [Codebook] DTD version history (via the Internet Archive Wayback Machine): <http://web.archive.org/web/20060711204401/http:/ www.icpsr.umich.edu/DDI/dtd/version-history.html> (Accessed February 12, 2014)

DDI Codebook Version 2.5 (XML schemas). <http://www.ddialliance. org/Specification/DDI-Codebook/2.5/>

DDI Controlled Vocabularies. <http://www.ddialliance.org/controlledvocabularies> (Accessed February 12, 2014)

DDI Lifecyle Model. (2004) <http://www.ddialliance.org/system/files/ Concept-Model-WD.pdf> (Accessed February 12, 2014)

DDI Lifecycle Version 3. <http://downloads.sourceforge.net/ddialliance/DDI_3_0_2008-04-28_Documentation_XMLSchema. zip?modtime $=1209367396>($ Accessed February 12, 2014)

DDI Lifecycle Version 3.1. <http://www.ddialliance.org/Specification/ DDI-Lifecycle/3.1/>

DDI Tools Catalog: <http://www.ddialliance.org/resources/tools $>$ (Accessed February 12, 2014)

DDI RDF Discovery and XKOS vocabularies. <http://www.ddialliance. org/Specification/RDF> (Accessed February 12, 2014)

European DDI Users Conference (EDDI) (Bonn, Germany), 2009. <http:// www.iza.org/conference_files/eddi09/2011_01_17_online_program. pdf> (Accessed February 12, 2014)

European DDI Users Conference (EDDI) (Utrecht, Netherlands), 2010. <http://www.iza.org/conference_files/eddi10/Program_2010-12-04_ JW.pdf> (Accessed February 12, 2014)

European DDI Users Conference (EDDI) (Gothenburg, Sweden), 2011 <http://www.iza.org/conference_files/EDDI2011/call_for_papers/ EDDI11_Program_2011-11-26.pdf> (Accessed February 12, 2014)

European DDI Users Conference (EDDI) (Bergen, Norway), 2012. <http://www.eddi-conferences.eu/ocs/public/conferences/1/ schedConfs/1/program-en_US.pdf> (Accessed February 12, 2014)

European DDI Users Conference (EDDI) (Paris, France), 2013.<http:// www.eddi-conferences.eu/ocs/index.php/eddi/EDDI13/schedConf/ program $>$ (Accessed February 12, 2014)

Inter-university Consortium for Political and Social Research (ICPSR). (2000) Final Report to National Science Foundation "Electronic Preservation of Data Documentation: Complementary

SGML and Image Capture" SBR-9617813 [August 1 1997-July 31, 2000] <http://www.ddialliance.org/sites/default/files/final.pdf>

Inter-university Consortium for Political and Social Research (ICPSR). (2001) Addendum to NSF Final Report "Electronic Preservation of Data Documentation: Complementary SGML and Image Capture," SBR-9617813: Results of the Evaluation of the Data Documentation Initiative (DDI), April 24, 2001. <http://www.ddialliance.org/sites/ default/files/evalsummary.pdf>

Minutes of Final Meeting of Original DDI Committee (Washington, DC), 2003: <http://www.ddialliance.org/DDI/committee-info/ minutes/2003-02-07.html> (Accessed February 12, 2014) (Accessed February 12, 2014)

Minutes of First Meeting of DDI Alliance Expert Committee (Ann Arbor, Ml), 2003. <http://www.ddialliance.org/DDI/committee-info/ minutes/2003-10-12.html> (Accessed February 12, 2014)

Minutes of DDI Expert Committee Meeting (Madison, WI), 2004. <http://www.ddialliance.org/DDI/committee-info/ minutes/2004-05-29.html> (Accessed February 12, 2014) 
Minutes of DDI Expert Committee Meeting (Edinburgh, Scotland), 2005. <http://www.ddialliance.org/DDI/committee-info/ minutes/2005-05-22.html> (Accessed February 12, 2014)

Minutes of DDI Expert Committee Meeting (Ann Arbor, MI), 2006. <http://www.ddialliance.org/DDI/committee-info/ minutes/2006-05-27.html> (Accessed February 12, 2014)

Minutes of DDI Expert Committee Meeting (Montreal, Canada), 2007. <http://www.ddialliance.org/DDI/committee-info/ minutes/2007-05-19.html> (Accessed February 12, 2014)

Minutes of DDI Expert Committee Meeting (Palo Alto, CA), 2008. <http://www.ddialliance.org/DDI/committee-info/ minutes/2008-05-31.html> (Accessed February 12, 2014)

Minutes of DDI Expert Committee Meeting (Tampere, Finland), 2009. $<$ http://www.ddialliance.org/sites/default/files/minutes/2009-05-25. pdf $>$ (Accessed February 12, 2014)

Minutes of DDI Expert Committee Meeting (Ithaca, NY), 2010. <http:// www.ddialliance.org/sites/default/files/DDI\%20Alliance\%20 Expert\%20Committee\%20Meeting\%20Minutes(2)\%205-31-2010. pdf> (Accessed February 12, 2014)

Minutes of DDI Expert Committee Meeting (Vancouver, Canada), 2011. <http://www.ddialliance.org/sites/default/files/ DDIAllianceExpertCommitteeMeetingMinutes2011-05-30_0.pdf> (Accessed February 12, 2014)

Minutes of DDI Expert Committee Meeting (Washington, DC), 2012. <http://www.ddialliance.org/sites/default/files/ DDIAllianceExpertCommitteeMeetingMinutes2012-06-04.pdf> (Accessed February 12, 2014)

Minutes of DDI Membership/Scientific Board (Cologne, Germany), 2013. $<$ http://www.ddialliance.org/system/files/DDIAllianceAnnual\%20 MeetingOfMembersAndScientificBoardMinutes2013-05-27.pdf> (Accessed February 12, 2014)

Minutes of DDI Executive Board. <http://www.ddialliance.org/alliance/ minutes $>$ (Accessed February 12, 2014)

Minutes of DDI Steering Committee. <http://www.ddialliance.org/ alliance/minutes > (Accessed February 12, 2014)

Minutes and Reports of DDI Developers Group. <http://www. ddialliance.org/alliance/working-groups\#ddc> (Accessed February 12, 2014)

Nielsen, Per (1974). "Report on Standardization of Study Description Schemes and Classification of Indicators." Association for Computing Machinery (ACM) SIGSOC Bulletin, Volume 6, Issue 2-3, Fall-Winter 1974-1975, pages 39-46. <http://dx.doi. org/10.1145/1102974.1102978>

North American DDI Users Conference (NADDI) (Lawrence, KS), 2013. $<$ http://www.ipsr.ku.edu/naddi/program.shtml> (Accessed February 12, 2014)

Participants in 2012 Dagstuhl Seminar on DDI Moving Forward. (2012) “Developing a Model-Driven DDI Specification." DDI Working Paper Series, Paper No. 4.

<http://dx.doi.org/10.3886/DDIWorkingPaper04>

Schloss Dagstuhl Workshop (First Dagstuhl Training) (Wadern, Germany), 2007. http://www.dagstuhl.de/en/program/calendar/ evhp/?semnr=07432 (Accessed February 12, 2014)

Schloss Dagstuhl Workshop on DDI Model (Wadern, Germany), 2013. <https://www.dagstuhl.de/en/program/calendar/ evhp/?semnr=12432> (Accessed February 12, 2014)

"Sprints" to Develop Model-based DDI. <http://www.ddialliance.org/ ddi-moving-forward-process> (Accessed February 12, 2014)

\section{Notes}

1. Mary Vardigan is an Assistant Director at the Inter-university Consortium for Political and Social Research (ICPSR) and Director of the DDI Alliance. She can be reached at vardigan@umich.edu.

2. Members of original SGML Codebook Committee:

- Merrill Shanks, UC-Berkeley, Chair

- Richard Rockwell, ICPSR

- Atle Alvheim, NSD

- Martin Appel, Census

- David Barber, Michigan

- Grant Blank, University of Chicago

- Bill Bradley, Health Canada

- Pat Doyle, AHCPR

- Terry Finnegan, National Center for Supercomputing Applications, University of Illinois

- Peter Granda, ICPSR

- Ann Green, Yale

- Stephan Greene, Maryland

- Lynn Jacobsen, Columbia

- John Price-Wilkin, Michigan

- Karsten Rasmussen, DDA

- Rolf Uher, Zentralarchiv, Koeln

- Mary Vardigan, ICPSR

3. Full URLs for all material linked in the Time Line can be found in References. 


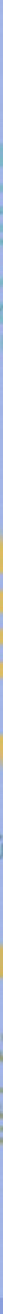

